\title{
Recovery from early cortical damage in rats: 5. Unilateral lesions have different behavioral and anatomical effects than bilateral lesions
}

\author{
BRYAN KOLB, JOANNE ZABOROWSKI, and IAN Q. WHISHAW \\ University of Lethbridge, Lethbridge, Alberta, Canada
}

\begin{abstract}
Rats with unilateral or bilateral removal of the cortex anterior to the bregma (frontal cortex) in adulthood were compared behaviorally and neuroanatomically to (1) rats with similar removals at 1 or 10 days of age, (2) rats with staged bilateral removals having a unilateral frontal lesion at 1 day and a second lesion at 10 days of age, and (3) rats that were hemidecorticated at 1 day of age. Age at lesion, unilaterality of lesion, and size of lesion interacted to alter brain development and behavior in different ways. Bilateral frontal lesions at 1 day of age produced a very small brain and thin cortex, and were associated with poor spatial learning. Similar removals at 10 days postnatal allowed sparing of spatial learning, but the cortex was still thinner than normal. Unilateral frontal lesions at any age produced similar behavioral effects, which were smaller than after bilateral lesions, and the neonatal lesions produced thinning of the ipsilateral cortex. Staged lesions allowed partial sparing of behavior, but there was cortical thinning equivalent to that after 1- or 10-day lesions in the respective hemispheres. In contrast to the effect of unilateral neonatal frontal lesions, which have little effect on the contralateral cortex, neonatal hemidecortication increased cortical thickness in the opposite hemisphere. These results imply that the effects of cortical injury in infancy vary with age, unilaterality of the lesion, and lesion size.
\end{abstract}

Cortical injury in infants can alter the course of their functional and anatomical development. For example, we have found that cortical damage leads to the development of a brain that is smaller, lighter in weight, and has thinner remaining cortex if the injury is sustained during the first week of life than if it is sustained in adulthood. Furthermore, the behavioral consequences of these early lesions are more severe than those of lesions in adulthood (e.g., Kolb, 1987; Kolb \& Holmes, 1983; Kolb, Sutherland, \& Whishaw, 1983a; Kolb \& Whishaw, 1981).

These results would seem to contradict a significant body of literature, which dates back to the influential studies of Kennard in the 1930s, showing that neonatal lesions are often associated with a better behavioral outcome than are lesions later in life (e.g., Hicks \& D'Amato, 1970; Kennard, 1938; Kolb \& Nonneman, 1976; Villablanca, Burgess, \& Sonnier, 1984). There appear to be at least two factors that may account for this discrepancy. First, we have recently found that the precise age at injury may lead to marked differences in the outcome of early lesions. Thus, damage to the frontal or parietal cortex at 10 days of life appears to allow sparing of function in adulthood, whereas similar damage in the first 5 days of life does not (Kolb, 1987; Kolb, Holmes, \& Whishaw,

This research was supported by grants from the Natural Sciences and Engineering Research Council of Canada to B.K. and I.W. The authors thank Brigitte Byers and Robbin Gibb for their help in behavioral and histological procedures. Correspondence may be addressed to Bryan Kolb, Department of Psychology, University of Lethbridge, Lethbridge, Alberta T1K 3M4, Canada.
1987). Some of the inconsistencies in the literature might therefore be related to what would appear to be rather small differences in the age at injury. Second, there is reason to suspect that the outcome of unilateral and bilateral lesions in infancy may differ markedly. Thus, there are numerous studies showing that restricted unilateral lesions of the sensorimotor cortex of various species may allow a better behavioral outcome than do similar lesions in adulthood (e.g., Hicks \& D'Amato, 1975; Kartje-Tillotson, Neafsey, \& Castro, 1985; Whishaw \& Kolb, 1988). Similarly, studies of the behavioral effects of neonatal hemidecortication show that the behavioral losses may be lessened if the lesions are performed in infancy (e.g., Kolb, Sutherland, \& Whishaw, 1983b; Villablanca et al., 1984).

Thus, it appears that both precise developmental age and unilaterality of cortical lesion might be significant factors influencing the outcome of early cortical lesions. The goal of the present experiment, therefore, was to compare the effects of unilateral and bilateral frontal cortical lesions in infant rats on adult cortical morphology and the performance of a spatial navigation task. In addition, we investigated whether staged bilateral neonatal lesions might allow greater sparing and less severe morphological changes than a single-stage bilateral lesion.

\section{METHOD}

\section{Subjects}

The animals were 103 Long-Evans rats divided into 10 groups: (1) 17 rats (10 female, 7 male) had their frontal cortex removed 
bilaterally on the day of birth (D1), (2) 12 rats (6 male, 6 female) had their frontal cortex removed unilaterally on D1 (6 left, 6 right), (3) 5 male rats had their frontal cortex removed bilaterally on Day 90 (D90), (4) 5 male rats had their frontal cortex removed unilaterally on D90 (3 left, 2 right), (5) 18 rats ( 12 male, 6 female) served as controls for the first four groups, (6) 10 rats $(5$ male, 5 female) were hemidecorticated on D1, (7) 6 male littermates served as controls, ( 8 ) 10 rats ( 5 female, 5 male) were given staged lesions, the frontal cortex of the right hemisphere being removed on D1 and the frontal cortex of the left hemisphere on D10, (9) 10 rats ( 5 male, 5 female) had their frontal cortex removed bilaterally on D1; and (10) 10 rats ( 5 male, 5 female) had bilateral frontal removals on D10. Behavioral testing occurred when the rats were 150-180 days of age. Since there were no sex differences in behavior, the data for males and females were combined. The animals were maintained on a 12:12 h light:dark cycle and were tested during the light cycle.

\section{Surgery and Anatomy}

Cortical tissue was removed by aspiration (Kolb \& Whishaw, 1981). Anesthesia was by cooling for the infants and sodium pentobarbital $(65 \mathrm{mg} / \mathrm{kg})$ for the adults. At the conclusion of the behavioral testing, the rats were deeply anesthetized and intracardially perfused with a solution of $0.9 \% \mathrm{NaCl}$. The neonatal frontal operates and all but 6 male controls were then perfused with a 10\% formalin solution. The brains were then removed, weighed, and cryoprotected in a $30 \%$ sucrose formalin solution, cut frozen at $40 \mu \mathrm{m}$, mounted, and stained with cresyl violet. The brains of the remaining controls and the rats with adult frontal lesions were processed for Golgi-Cox staining, the results of which are reported elsewhere (Kolb \& Gibb, 1987). The brains of the hemidecorticates and their controls were perfused with $0.9 \%$ saline and then a $10 \%$ formalin solution before being processed for embedding in celloidin. The frozen sections were cut at $40 \mu$ and the Golgi sections at $120 \mu$. Cortical thickness measurements were made using procedures described by Kolb (1987). Three measurements were made at each of five planes corresponding to Paxinos and Watson's (1986) Plates 12, 18, 27, 37, and 44.

\section{Spatial Navigation}

The spatial navigation test required the rats to find a hidden platform in a swimming pool (Sutherland, Whishaw, \& Kolb, 1983). The pool was a circular tank ( $85 \mathrm{~cm}$ diam; $45 \mathrm{~cm}$ high), the inside of which was painted white, and was filled to a height of $25 \mathrm{~cm}$ with approximately $18^{\circ} \mathrm{C}$ water in which $1,000 \mathrm{cc}$ of instant powdered skim milk was dissolved. A clear Plexiglas platform $(11 \times 12 \mathrm{~cm})$ was present inside the pool; its top surface was $1 \mathrm{~cm}$ below the surface of the water.

A trial consisted of placing a rat by hand into the water, facing the wall of the pool, at one of four equally spaced starting locations around the pool's perimeter. Within each block of four trials, each rat started at the four starting locations, but the sequence of locations was randomly selected.

Behavioral testing was conducted on 5 consecutive days, with each rat receiving eight trials per day. If, on a particular trial, the rat found the platform, it was permitted to remain there for $10 \mathrm{sec}$. A trial was terminated if the rat failed to find the platform after $90 \mathrm{sec}$. At the end of a trial, the rat was returned to a holding cage, and approximately $5 \mathrm{~min}$ elapsed before beginning the next trial. The swimming path for each trial was recorded on a map of the pool by an experimenter seated by the pool's edge. The latency to find the platform was timed for each trial. There was a small modification in the testing procedure for the rats in the staged-lesion study. These rats received nine trial blocks as described, but on the 10th trial block, the platform was moved to the opposite side of the pool. This constituted a probe trial to determine if the animals had learned the location of the platform relative to distal room cues, or had sim- ply learned a strategy of effectively searching the pool to find it. In the former case, there should be an increase in latency, since the platform was in a new location, but in the latter case, moving the platform should be without effect.

\section{ANATOMICAL RESULTS}

\section{Gross Morphology}

The neonatal frontal lesions produced visually smaller hemispheres ipsilateral to the damage, the D1 lesions producing the smallest hemispheres. In the rats with unilateral lesions, the contralateral hemisphere appeared normal in size, as it did in the hemidecorticates. The contrasting effect of the D1 and D10 lesions was clearly visible in the rats with staged lesions: the D1 lesion led to the development of a much smaller hemisphere than did the D10 lesion. One of the principal reasons for the smaller hemispheres after early frontal lesions was that the cortex was thinner in the neonates, as shown in Figures 1 and 2 . The reduction in brain size was quantified by measuring brain weights and cortical thickness, which we shall consider separately below.

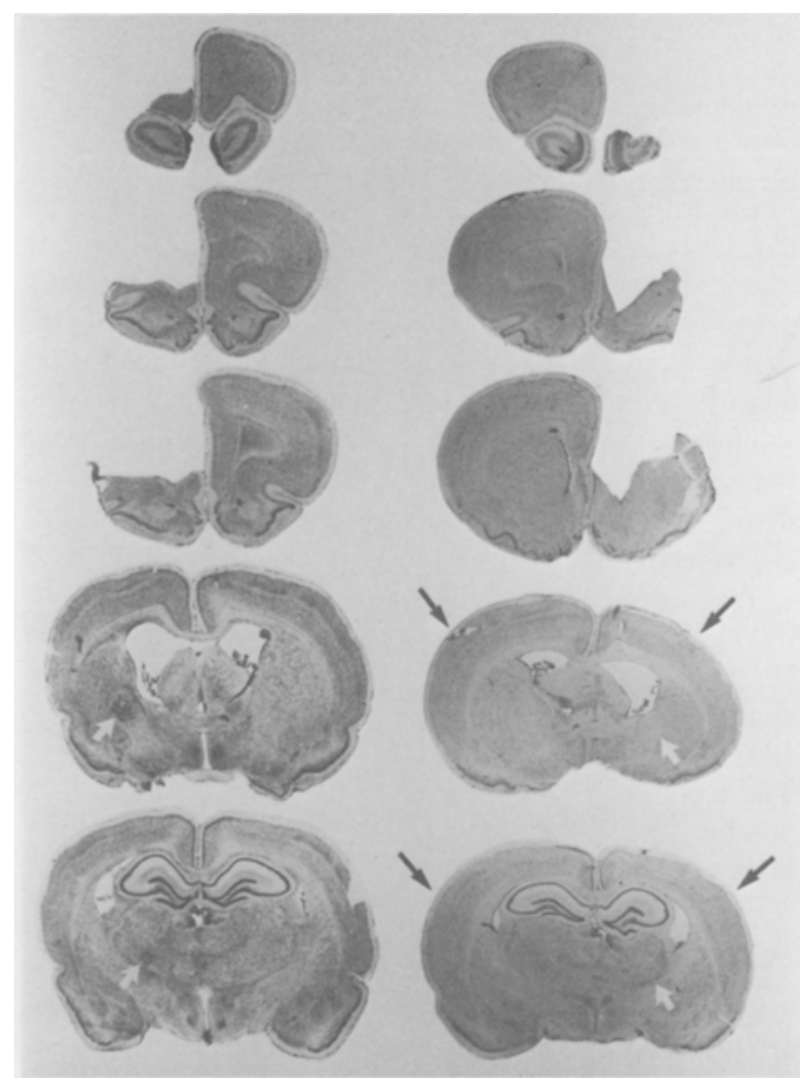

Figure 1. Serial sections through a representative adult (left) and neonatal (right) unilateral frontal operate. The black arrows point to the obvious difference in cortical thickness in the neonatal operate. The white arrows point to the clear calcification of the globus pallidus in the adult operate, which is not seen in the neonatal operate, and to the calcification in the thalamus of the adult but not the neonatal operate. Cortical thickness in the adult and neonatal rats cannot be compared directly in the figure because fixation times differed. 


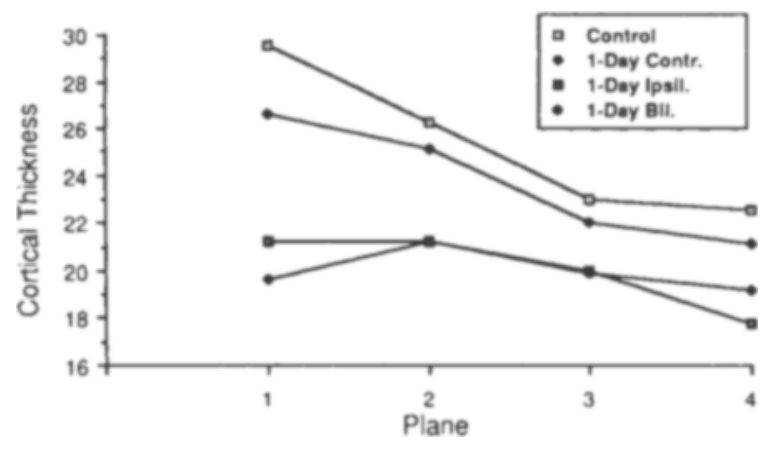

Figure 2. Summary of cortical thickness measurements at different planes of section posterior to the frontal lesions. The cortex is thinner throughout the entire anterior-posterior extent of the hemisphere.

\section{Retrograde Changes}

The adult frontal lesions resulted in areas of gliosis and calcification (see Whishaw, Schallert, \& Kolb, 1981) in the ipsilateral globus pallidus and thalamus, as shown in Figure 1. Neither gliosis nor calcification were observed in the neonatal operates, but the thalamus was shrunken as we have reported elsewhere (Kolb, Sutherland, \& Whishaw, 1983a) and as illustrated in Figure 1.

\section{Brain Weight}

Frontal lesions. Bilateral neonatal lesions had a greater effect upon brain weight than did adult lesions, whereas unilateral lesions in infants and adults had more similar effects relative to control weight (see Table 1). An analysis of variance (ANOVA) on the brain weight of neonatal operates revealed a significant effect of group for both the males $[F(2,16)=10.7, p<.001]$ and the females $[F(2,19)=48.2, p<.001]$. Follow-up tests (Tukey's $a$ ) found the brains with bilateral lesions to be smaller than those with unilateral lesions, which in turn were smaller than the controls' ( $p$ s $<.01$ ). The analysis on the adult operates also yielded significant results $[F(2,14)=7.69, p<.01]$. Follow-up tests showed the controls to have heavier brains than the two frontal groups' $(p s<.01)$, which did not differ significantly $(p>.05)$. There was no overlap in the brain weights of the comparable adult and neonatal groups, the adults having the larger brains.
Staged lesions. The male rats with staged lesions had brains that were equivalent in size to those of D10 operates, whereas the female rats with staged lesions had smaller brains, being equivalent to female rats with D1 lesions (Table 1). ANOVAs comparing the control- $(n=$ 5), D1- $(n=5)$, D10- $(n=5)$, and staged-lesion $(n=$ 5) animals showed significant main effects for both the males $[F(3,18)=62.4, p<.001]$ and the females $[F(3,19)=20.4, p<.001]$. Post hoc tests showed the sex difference to be reliable: the males with staged lesions differed from the control and D1 groups, but not from the D10 group, whereas the females differed from the control and D10 groups ( $p s<.05$ or better).

Hemidecortications. The neonatal hemidecorticates had brains that weighed about $80 \%$ of those of control. There was no overlap in the lesion and control groups $(p<.001)$.

\section{Cortical Thickness}

Frontal lesions. Adult and infant lesions had different effects on the thickness of the remaining cortex (Figure 2). Adult lesions had little effect distal to the lesion, either ipsilaterally or contralaterally. In contrast, bilateral lesions in infancy produced a large decrease in cortical thickness throughout the remaining hemisphere, and unilateral lesions had a correspondingly large effect ipsilaterally. In addition, the unilateral lesions had a small, but statistically reliable, effect contralaterally, as illustrated in Figure 2. Owing to differences in fixation procedures, the cortical thickness in the adult and the neonatal groups had to be analyzed separately. ANOVAs were done on the groups (control, bilateral, unilateral), plane of measurement, and side of brain.

The ANOVA on the infants and their appropriate controls showed a main effect of group $[F(2,36)=162$, $p<.001]$, plane $[F(4,144)=189.4, p<.001]$, and side $[F(1,36)=80.7, p<.001]$. In addition, all of the possible interactions were significant $(p s<.001)$. Post hoc tests (Tukey's $a$ ) found that (1) the animals with bilateral lesions differed from the controls, (2) the animals with unilateral lesions had thinner cortex on the lesion side, and (3) the contralateral cortex of the unilateral operates was thinner than that of the controls ( $p s<.05$ or better).

The ANOVA on the adult brains revealed a main effect of group $[F(2,12)=136, p<.001]$, plane $[F(4,48)$

Table 1

Summary of Brain Weights (in grams)

\begin{tabular}{|c|c|c|c|c|c|c|}
\hline \multirow[b]{2}{*}{ Control } & \multicolumn{3}{|c|}{ Bilateral Lesions } & \multicolumn{2}{|c|}{ Unilateral Lesions } & \multirow[b]{2}{*}{ Neonatal Staged } \\
\hline & Adult Frontal & Day 1 Frontal & Day 10 Frontal & Adult Frontal & Day 1 Frontal & \\
\hline \multicolumn{7}{|c|}{ Male } \\
\hline $2.103 \pm .014$ & $1.805 \pm .071$ & $1.528 \pm .049$ & $1.695 \pm .029$ & $1.992 \pm .040$ & $1.891 \pm .046$ & $1.639 \pm .018$ \\
\hline \multicolumn{7}{|c|}{ Female } \\
\hline $1.959 \pm .040$ & & $1.514 \pm .035$ & $1.675 \pm .042$ & & $1.755 \pm .020$ & $1.511 \pm .018$ \\
\hline
\end{tabular}

Note-Data refer to means and standard errors. For the male rats with bilateral neonatal frontal lesions, the Day 1 bilateral group is smaller than all others $(p<.05$ or better) and the Day 10 and staged groups do not differ. For the female rats, the Day 1 bilateral group is equivalent to the staged group and both are smaller than the Day 10 group ( $p s<.05$ or better). 


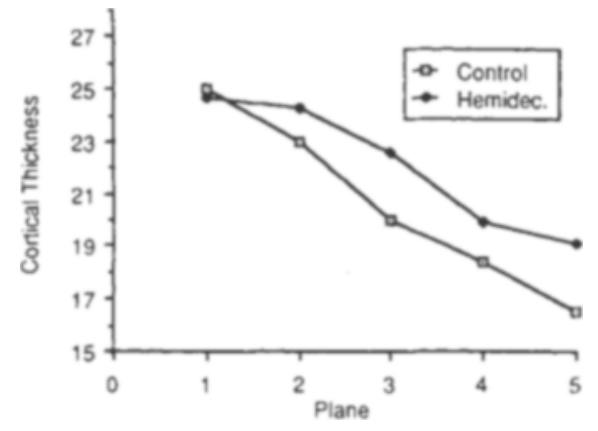

Figure 3. Effects of neonatal hemidecortication on cortical thickness at five planes sectioned on the side contralateral to the lesion.

$=310.5, p<.001]$, and hemisphere $[F(1,12)=282.2$, $p<.001]$. All of the possible interactions were also significant $(p s<.001)$. Post hoc tests $(p<.05)$ showed the cortex to be thinner in the lesion groups in the first plane behind the lesions (Plane 2), but at no other plane $(p<.05)$. In contrast to the neonatal group, the unilateral adults had a contralateral cortex of normal thickness (see Figure 3).

Staged lesions. Staged removal of the frontal cortex resulted in changes in cortical thickness in each hemisphere that were identical to those following the respective bilateral lesions. Thus, the hemisphere with a D1 removal had a cortical thickness equivalent to that in rats with bilateral D1 lesions, whereas the hemisphere with a D10 removal had a thickness similar to that of rats with bilateral D10 lesions.

A repeated-measures ANOVA with hemisphere and plane of measure as factors compared the two hemispheres of the control rats to those of the staged-lesion rats. The results yielded significant effects for group $[F(1,10)=$ $4.38, p=.06]$, plane $[F(3,30)=229.6, p<.001]$, and side $[F(1,10)=21.5, p<.001]$, as well as significant group $\times$ plane $[F(3,30)=9.28, p<.001]$, group $\times$ side $[F(1,10)=16.74, p<.001]$, plane $\times$ side $[F(3,30)$ $=3.97, p=.02]$, and group $\times$ plane $\times$ side $[F(3,30)$ $=5.19, p<.001]$. Post hoc tests (Tukey's $a$ ) allow us to conclude that the hemisphere with the D1 lesion was thinner at every plane than the hemisphere with the D10 lesion, which was thinner than the control hemispheres at every plane.

Hemidecortication. In contrast to the effect of neonatal removal of the frontal cortex, removal of an entire hemisphere resulted in an increase in cortical thickness in the remaining hemisphere, relative to control values (see Figure 3). An ANOVA (with group and plane as factors) revealed a significant effect of group $[F(1,17)=22.5$, $p<.001]$ and plane $[F(4,68)=92.9, p<.001]$, as well as the interaction of the two $[F(4,68)=4.58, p<.01]$. It can be seen in Figure 3 that the increase in cortical thickness was progressively greater moving from front to back in the brains, which is reflected in the significant interaction.

\section{BEHAVIORAL RESULTS}

When initially placed in the tank, normal control rats traverse a wide area, zig-zagging across the tank until they bump into the hidden platform. Their performance improves rapidly on successive trials, and within about 12 trials (three blocks of four trials), they have reached asymptote, swimming directly to the platform from any start location in about $5 \mathrm{sec}$.

\section{Effects of Frontal Lesions}

It can be seen in Figure 4 that rats with large bilateral frontal lesions have a large performance deficit, as they initially performed poorly on the task and improved slowly. Furthermore, there was a clear difference between the adult and the neonatal operates; the neonates failed to improve below a latency of about $30 \mathrm{sec}$ by the end of training. Inspection of the swim paths showed that this was because they never learned to swim directly to the platform but, rather, swam randomly around the tank until bumping into the platform. Thus, their improvement

A. Neonatal Lesions

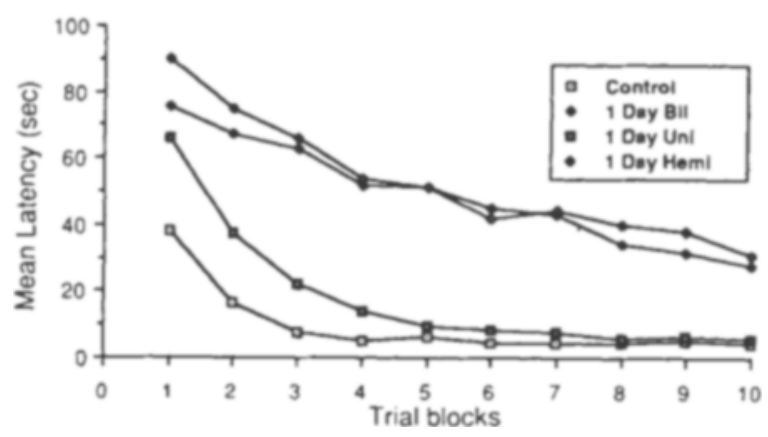

B. Adult Lesions

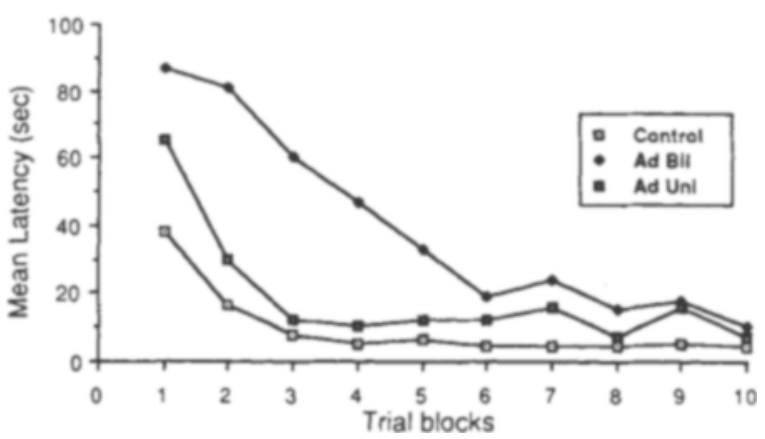

Figure 4. Summary of the latency for rats to find the submerged platform following neonatal or adult lesions. Neonatal hemidecortication or bilateral frontal removal produce severe performance deficits, whereas unilateral lesions at 1 day of age produce only a small behavioral impairment. Similarly, in adulthood, unilateral lesions produce a small behavioral deficit, although the effect of bilateral lesions in adulthood is less than that of similar lesions at 1 day of age. 
largely reflects their learning to leave the wall of the tank in search of the platform, as we have analyzed in detail elsewhere (Kolb, 1987). In contrast, rats with unilateral frontal lesions learned relatively quickly, although they were less consistent in their performance than were normal rats.

An ANOVA found significant main effects of group $[F(4,39)=24.6, p<.001]$ and trials $[F(9,342)=40.3$, $p<.001]$, and a significant interaction $[F(36,342)=2.6$, $p<.001]$. Follow-up comparisons (Tukey's $a, p s<.05$ or better) showed the bilateral groups' performance to be significantly worse overall than the unilateral groups', and the neonatal bilateral frontals' performance to be worse overall than the adult bilateral frontals'. The two unilateral groups did not differ.

\section{Effects of Hemidecortication}

The hemidecorticate rats were poor at the water task, performing virtually identically to the D1 bilateral frontals (Figure 4). Group differences were significant between the control and the hemidecorticates and between the hemidecorticates and the D1 unilateral frontals after the first trial block ( $p s<.05$ or better), but not between the hemidecorticate and the D1 bilateral groups ( $p s>.10)$.

\section{Effects of Staged Lesions in Infancy}

In view of the contrasting effects of unilateral and bilateral frontal lesions in infancy, we had expected some advantage in making staged unilateral frontal lesions in infancy. Figure 5 summarizes the performance of rats with bilateral lesions at D1 or D10 and of rats with unilateral frontal lesions at D1, followed by another such lesion at D10. It can be seen that although the rats with staged lesions were superior to those with lesions at D1, the animals were severely impaired relative to animals with lesions at D10. Indeed, the staged-lesion group was still taking more than twice as long to locate the platform on the ninth trial block than were the control and D10 frontal groups. Moreover, whereas the latter groups

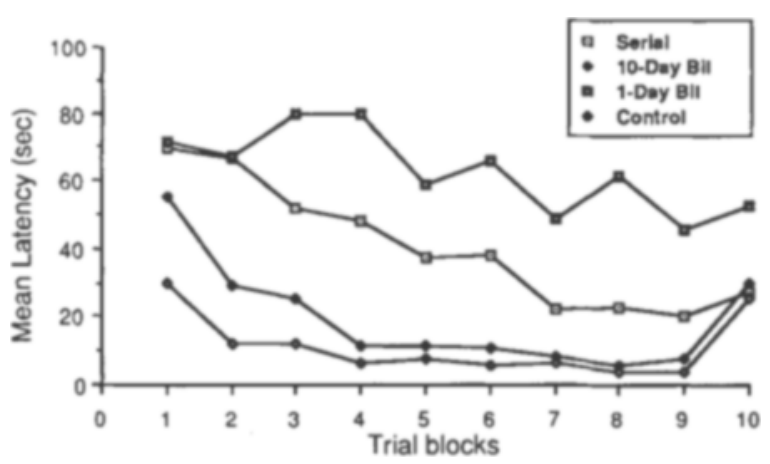

Figure 5. Summary of the latency to find the submerged platform for rats with bilateral lesions in infancy performed at 1 day of age, 10 days of age, or serially at 1 and then at 10 days of age. showed a clear increase in latency on the reversal trials (Block 10), staged lesions produced little increase.

An ANOVA found significant main effects of lesion group $[F(3,324)=24.63, p<.001]$ and trial block $[F(9,324)=23.23, p<.001]$, and a significant group $\times$ trial block interaction $[F(27,324)=3.78, p<.001]$. Tukey's $(a)$ post hoc tests showed that the staged-lesion group differed from the control and D10 groups on every trial block except Block 10 (reversal block), and differed from the D1 group on Blocks 3-10. Furthermore, whereas the D10 and control groups showed significant increases on the reversal block, none of the other groups did. Finally, the D10 group differed from the control group only on Blocks 1-3.

\section{DISCUSSION}

Rats with bilateral frontal lesions on D1 had severe impairments in spatial navigation, very small brains, and thin posterior cortex, relative to adults. In contrast, rats with unilateral frontal lesions in infancy had a comparatively smaller behavioral disruption, but significant thinning of the ipsilateral cortex, relative to adult operates. Furthermore, although hemidecortication produced an increase in contralateral cortical thickness, no parallel change $o c-$ curred after unilateral frontal lesions. Finally, staged cortical lesions led to changes in cortical thickness equivalent to those observed in the respective D1 and D10 groups, and allowed only partial sparing of function. These results led us to several conclusions.

First, damaging the cortex at birth in rats has severe effects upon the anatomical development of the rest of the cortex and on subsequent behavior. The reduction in brain weight and cortical thickness, along with the complete absence of sparing from early lesions, is consistent with the emerging view that early neural injury can have effects as great or greater than those of later neural injury (e.g., Goldman \& Galkin, 1978; Gramsbergen \& IjkemaPassen, 1984; Hicks \& D'Amato, 1975; Isaacson, 1975; Kolb, 1987).

Second, the results confirm the suggestion that the restriction of brain damage to one hemisphere is a powerful factor in favor of recovery (Olmstead \& Villablanca, 1979; Villablanca et al., 1984; Whishaw \& Kolb, 1988). Indeed, animals with unilateral removals of the entire frontal cortex appear to be more normal behaviorally than do animals with small bilateral removals of the medial frontal cortex or motor cortex in which less total tissue is removed (Kolb \& Whishaw, 1985a, 1985b).

Third, the comparatively less severe effects of unilateral lesions in infancy supports the idea that recovery is more likely to occur if part of a functional system is still intact (in this case, the cortex in the opposite hemisphere) than if recovery is to be mediated by damaged systems (e.g., remaining frontal cortex following small bilateral lesions) or by systems that do not normally mediate particular 
functions (e.g., parietal cortex in the absence of frontal cortex). The reason why an intact system may allow better behavioral sparing or recovery may be that anatomical reorganization is more likely or more extensive in intact systems.

Fourth, rats with unilateral frontal lesions early in infancy appear to fare better than human infants with similar damage, in spite of the abnormal development of the ipsilateral hemisphere. One reason for this may be that the two hemispheres of the rat are more similar in function than they are in humans. Thus, damage in rats may still leave an intact contralateral system capable of mediating at least some behaviors. Alternatively, it may be that we are not assessing comparable behavioral effects in our rats and in humans. Had we administered a more general test battery to the rats, we might have found a general loss of efficiency in behavior, as we have found previously for rats with small bilateral lesions in infancy (Kolb $\&$ Whishaw, 1985b). Furthermore, we have found that although small motor cortex lesions in rats allow substantial sparing of reaching in the contralateral paw, there is a corresponding loss of efficiency with the ipsilateral paw (Whishaw \& Kolb, 1988), which also may suggest a general effect on behavior of the infant rat with unilateral injury. Future studies will need to include a more extensive behavioral analysis of rats with unilateral lesions.

Finally, the current anatomical data imply that the thicker contralateral cortex observed after hemidecortication does not occur after partial cortical lesions. We believe the increase in cortical thickness in hemidecorticates to be reliable, since we have found it in four different experiments (current study; Kolb et al., 1983a, 1983b; Kolb \& Tomie, 1988), but we still do not understand its basis. It has been shown by several groups that there are numerous abnormal corticofugal connections from the intact hemisphere (e.g., Hicks \& D'Amato, 1975; Villablanca et al., 1984; Whishaw \& Kolb, 1988), and these may take up more space. Furthermore, in a parallel study, we have found that hemidecortication increases arborization of Layer II/III pyramidal cell basilar dendrites in many regions of the normal hemisphere, whereas small unilateral lesions at Postnatal Day 1 produce a retraction of the same dendrites ipsilaterally (Kolb \& Gibb, 1987) and produce no significant contralateral changes. Thus, the increased cortical thickness after hemidecortication may result from both an increase in corticofugal connections and increased dendritic arbor in the cortex. It remains to be shown if the changes in cortical structure are related directly to the anomalous connections.

\section{REFERENCES}

Goldman, P. S., \& Galkin, T. W. (1978). Prenatal removal of frontal association cortex in the fetal rhesus monkey: Anatomical and functional consequences in postnatal life. Brain Research, 152, 451-458. GramsBergen, A., \& IJKEMA-PASSEN, J. (1984). The effects of early cerebellar hemispherectomy in the rat: Behavioral, neuroanatomical, and electrophysiological sequelae. In S. Finger \& C. R. Almli (Eds.), Early brain damage (Vol. 2, pp. 155-178). New York: Academic Press.

Hicks, S. P., \& D'Amato, C. J. (1970). Motor-sensory and visual behavior after hemispherectomy in newborn and mature rats. Experimental Neurology, 29, 416-438.

Hicks, S. P., \& D'Amato, C. J. (1975). Motor-sensory cortex-corticospinal system and developing locomotion and placing in rats. American Journal of Anatomy, 143, 1-42.

ISAACSON, R. L. (1975). The myth of recovery from early brain damage. In N. E. Ellis (Ed.), Aberrant development in infancy (pp. 1-26). New York: Wiley.

Kartje-Tillotson, G., Neafsey, J. E. J., \& Castro, A. J. (1985). Electrophysiological analysis of motor cortical plasticity after cortical lesions in newborn rats. Brain Research, 332, 103-111.

KenNard, M. A. (1938). Reorganization of motor function in the cerebral cortex of monkeys deprived of motor and premotor areas in infancy. Journal of Neurophysiology, 1, 477-496.

KoLB, B. (1987). Recovery from early cortical damage in rats: 1. Differential behavioral and anatomical effects of frontal lesions at different ages of neural maturation. Behavioural Brain Research, 25 , 205-220.

KolB, B., GiBB, R. (1987). Dendritic proliferation as a mechanism of recovery and sparing of function. Society for Neuroscience Abstracts, $13,1430$.

KolB, B., Holmes, C. (1983). Neonatal motor cortex lesions in the rat: Absence of sparing of motor behaviors and impaired spatial learning concurrent with abnormal cerebral morphogenesis. Behavioral Neuroscience, 97, 697-709.

KolB, B., Holmes, C., \& Whishaw, I. Q. (1987). Recovery from early cortical lesions in rats: 3 . Neonatal removal of posterior parietal cortex has greater behavioral and anatomical effects than similar removals in adulthood. Behavioural Brain Research, 26, 119-137.

Kolb, B., \& Nonneman, A. J. (1976). Functional development of prefrontal cortex in rats continues into adolescence. Science, 193, 335-336.

Kolb, B., Sutherland, R. J., \& Whishaw, I. Q. (1983a). Abnormalities in cortical and subcortical morphology after neonatal neocortical lesions in rats. Experimental Neurology, 79, 223-244.

Kolb, B., Sutherland, R. J., * Whishaw, I. Q. (1983b). Neonatal hemidecortication or frontal cortex ablation produces similar behavioral sparing but opposite effects on morphogenesis of remaining cortex. Behavioral Neuroscience, 97, 154-158.

KOLB, B., \& TOMIE, J. (1988). Recovery from early cortical damage in rats. IV. Effects of hemidecortication at 1, 5, or 10 days of age on cerebral anatomy and behavior. Behavioural Brain Research, 28 , 259-284.

KolB, B., \& Whishaw, I. Q. (1981). Neonatal frontal lesions in the rat: Sparing of learned but not species-typical behavior in the presence of reduced brain weight and cortical thickness. Journal of Comparative \& Physiological Psychology, 95, 863-879.

KolB, B., \& Whishaw, I. Q. (1985a). Earlier is not always better: Behavioral dysfunction and abnormal cerebral morphogenesis following neonatal cortical lesions in the rat. Behavioural Brain Research, $17,25-43$.

Kolb, B., \& Whishaw, I. Q. (1985b). Neonatal frontal lesions in hamsters impair species-typical behaviors and reduce brain weight and neocortical thickness. Behavioral Neuroscience, 99, 691-706.

Olmstead, C. E., \&illablanca, J. R. (1979). Effects of caudate nuclei on frontal cortical ablations in kittens: Paw usage. Experimental Neurology, 69, 559-572.

PAXINOS, G., \& WATSON, C. (1986). The rat brain in stereotaxic coordinates (2nd ed.). Orlando, FL: Academic Press.

SutherLand, R. J., WhISHAw, I. Q., \& KolB, B. (1983). A behavioural analysis of spatial localization following electrolytic, kainate- or colchicine-induced damage to the hippocampal formation in the rat. Behavioural Brain Research, 7, 133-153.

Villablanca, J. R., Burgess, J. W., \&onnier, B. J. (1984). Neo- 
natal cerebral hemispherectomy: A model for postlesion reorganization of the brain. In S. Finger \& C. R. Almli (Eds.), Recovery from brain damage (Vol. 2, pp. 179-210). New York: Academic Press. WhISHAW, I. Q., KolB, B. (1988). Sparing of skilled forelimb reaching and corticospinal projections after neonatal motor cortex removal or hemidecortication in the rat: Support for the Kennard doctrine. Brain Research, 451, 97-114.
Whishaw, I. Q., Schallert, T., \& Kolb, B. (1981). An analysis of feeding, sensory and motor abilities of rats after decortication. Journal of Comparative \& Physiological Psychology, 95, 85-103.

(Manuscript received July 19, 1988; revision accepted for publication May 5, 1989.) 